\title{
Polaritonic Microwave Waves of Inclined Incidence in Some Magnetodielectric Superlattices
}

\author{
J. KAPELEWSKI* \\ Faculty of Electronics, Military University of Technology \\ S. Kaliskiego 2, 00-908 Warsaw, Poland
}

\begin{abstract}
This paper concerns a theoretical study of microwave phonon-polaritons excited in a planar magneto-dielectric composite by a plane electromagnetic wave obliquely incident on its surface. The composite consists of alternately spaced piezoelectric and piezomagnetic layers having transverse isotropic properties for both electromagnetic and elastic parameters. Results include direction-dependent dispersion for phase velocity of phonon-polariton modes being excited by the incident electromagnetic wave in the superlattice in consideration here. A peculiar kind of coupling is proved to appear in the system treated, which do not exist in the case of electromagnetic waves propagating along principal symmetry axes. The paper concludes with a short review of available literature data on current and prospective applications of the microwave polaritons.
\end{abstract}

PACS: 41.20.Jb

\section{Introduction}

Study of the periodic media has long been a topic of interest. In a crystal, the periodic potential causes the energy of electrons to form a band structure with only electrons in passbands that are capable of moving freely. In artificial composites such as superlattices, the periodic modulation of selected physical parameters may also result in band structure and novel properties. Associated with the variation of permittivity (also permeability or both) are so-called photonic crystals, which are important for applications such as suppressing spontaneous emission, manipulating electromagnetic (EM) wave in a specific path and many others. Recently, also interest in phononic crystal, a periodic elastic superlattice, has grown. Attention has been given to phenomena, such as various sensors and actuators, acoustic filters, new transducers. If the piezomagnetic (as in some antiferromagnetic media) or piezoelectric coefficient is modulated in crystals of the kind, the interaction between the superlattice vibration and EM waves may arise, creating a kind of polariton excitation.

Peculiar kind of polaritons are so called phonon-polaritons. They can be regarded as collective excitations resulting from strong coupling of EM waves with an electric or magnetic dipole-carrying lattice with an axial symmetry (or superlattice) vibrations. In general, they describe the crossing of the EM dispersion with any interacting resonance. A major feature of polaritons is a sufficiently strong dependence of the propagation speed on the frequency in the resonance region.

\footnotetext{
* corresponding author; e-mail: jerzy.kapelewski@wat.edu.pl
}

The coupled polariton modes exhibit anticrossing in frequency with a level separation corresponding to vacuum-field Rabi splitting, in analogy to the atomic physics phenomenon.

Wave propagation in bulk media is determined by the dispersion relation of corresponding excitations, and the band-gap in excitation spectra is very useful in providing an "insulating background" for the modes within that frequency window. It offers the opportunity for local engineering of microstructure, the modes within the band-gap, by way of specially designed defect-doping. This plays an important role in guiding wave propagation in the medium, and paves the way for a number of device applications.

It is well known that, in an ionic crystal, the phonon-polariton originates from the coupling between lattice vibrations (transverse optical phonons) and EM waves (photons), resulting in the infrared absorption and dielectric abnormality, etc. In a piezoelectric superlattice the situation becomes more complicated because of the anisotropic nature of the piezoelectricity. There is not only the polariton driven by the transverse superlattice vibration, but also the polariton associated with the longitudinal superlattice vibration.

The research area of microwave photonics can be divided into two parts. First, the study of opto-electronic devices and systems processing signals at microwave rates. Second, the use of opto-electronic devices and systems for signal handling in microwave systems. Digital optical-fiber systems now carry the bulk of terrestrial long-distance communications traffic and fiber is increasingly being brought into the local access network. The use of opto-electronics in microwave systems has now become a commercial reality in fiber-radio access networks 
and there are emerging applications in phased-array antennas, electronic warfare, ultrafast noninvasive measurements, and even radio astronomy $[1,2]$.

As opposed to ionic (polar)-crystal phonon-polaritons, there is still rather few theoretical and experimental research on the phonon-polariton in superlattices, whose periodicity lies in the range of micrometers.

By solving the Maxwell equations for averaged dielectric and ferromagnetic medium we were able to show that magnetoelectric coupling can, in some conditions, effect in dramatic new polarization mode, having peculiar dispersion properties change of the polaritonic spectrum.

In the paper we present a theoretical discussion of phonon-polaritons for laminal superlattices, being a structure composed of alternating magneto-electric layers. The method includes homogenization of the material providing the description in terms of effective global parameters.

We will show that even large change in polaritonic spectra is possible due to magneto-electric (ME) coupling of oblique incidence EM wave with the medium in the composite in consideration.

\section{Formulation of the problem}

Let us consider now the following case. A piezoelectric-piezomagnetic superlattice is fabricated by alternated layers of piezoelectric (PE) and piezomagnetic (PM) materials arranged along $z$ axis. For simplification here, we assumed the PE and PM layers with the same thickness $d$ are polycrystalline and electronically and magnetically poled along the $z$ direction. So both PE and PM layers could be considered as the transversely isotropic systems with the same point group of $\infty m$ symmetry. Figure 1 is a schematic diagram of this case. The superlattice is also regarded as a complex structure made of a PE superlattice and a PM superlattice.

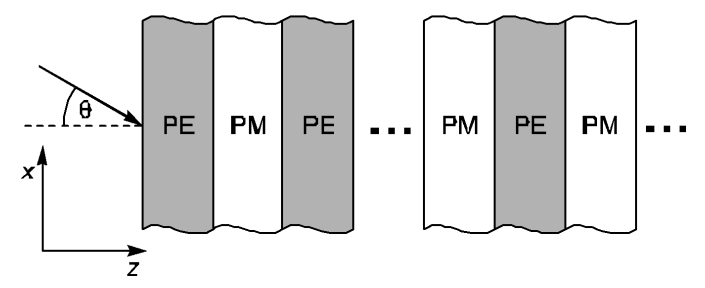

Fig. 1. Schematic of the piezoelectric-piezomagnetic superlattice.

The interaction between the EM wave and acoustic wave is described by the following equations (see e.g. $[3-6])$ :

$$
\begin{aligned}
& T_{I}=C_{I J} S_{J}(z)-e_{I j} \theta^{(\mathrm{e})}(z) E_{j}-q_{I j} \theta^{(\mathrm{m})} H_{j}, \\
& D_{i}=e_{i J} \theta^{(\mathrm{e})} S_{J}(z)+\varepsilon_{i j}(z) E_{j}+\alpha_{i j}(z) H_{j}, \\
& B_{i}=q_{i J} \theta^{(\mathrm{m})} S_{\mathrm{J}}(z)+\mu_{i j}(z) H_{j}+\beta_{i j}(z) E_{j} \\
& \quad(i, j=1,2,3 ; I, J=1,2, \ldots, 6) .
\end{aligned}
$$

For the effective permittivity and permeability, piezoelectric, piezomagnetic and elastic tensors of the average uniaxial superlattice for the uniaxial symmetry in the Voigt notation, we have

$$
\begin{aligned}
\boldsymbol{\varepsilon}= & {\left[\begin{array}{ccc}
\varepsilon_{\perp} & 0 & 0 \\
0 & \varepsilon_{\perp} & 0 \\
0 & 0 & \varepsilon_{\|}
\end{array}\right], \quad \boldsymbol{\mu}=\left[\begin{array}{cccc}
\mu_{\perp} & 0 & 0 \\
0 & \mu_{\perp} & 0 \\
0 & 0 & \mu_{\|}
\end{array}\right], } \\
\boldsymbol{d} & =\left[\begin{array}{cccccc}
0 & 0 & 0 & 0 & d_{15} & 0 \\
0 & 0 & 0 & d_{15} & 0 & 0 \\
d_{31} & d_{31} & d_{33} & 0 & 0 & 0
\end{array}\right], \quad \boldsymbol{d} \equiv \boldsymbol{e} \text { or } \boldsymbol{q}, \\
\boldsymbol{C}= & {\left[\begin{array}{cccccc}
C_{11} & C_{12} & C_{13} & 0 & 0 & 0 \\
C_{12} & C_{11} & C_{13} & 0 & 0 & 0 \\
C_{13} & C_{13} & C_{33} & 0 & 0 & 0 \\
0 & 0 & 0 & C_{44} & 0 & 0 \\
0 & 0 & 0 & 0 & C_{55} & 0 \\
0 & 0 & 0 & 0 & 0 & C_{66}
\end{array}\right] . }
\end{aligned}
$$

We also assume that the transverse dimensions are much larger than the acoustic wavelength in the superlattice. By using a 1D model and the long-wave approach, and substituting $T_{5}$ into the equation of motion for superlattice vibration, we can obtain

$$
\begin{aligned}
& \rho \frac{\partial^{2} S_{5}}{\partial t^{2}}-C_{55} \frac{\partial^{2} S_{5}}{\partial z^{2}} \\
& \quad=\frac{\partial^{2}}{\partial z^{2}}\left[-e_{15} \theta^{(\mathrm{e})}(z) E_{1}-q_{15} \theta^{(\mathrm{m})}(z) H_{1}\right] .
\end{aligned}
$$

Here $\rho$ and $C_{55}=C_{1331}$ are the effective mass density and the elastic coefficient, respectively. With the Fourier transformation, the modulation functions are written as

$$
\begin{aligned}
& \theta^{(\mathrm{e})}(z)=\sum_{n} \theta_{n}^{(\mathrm{e})} \mathrm{e}^{\mathrm{j} K_{n} z}, \\
& \theta^{(\mathrm{m})}(z)=\sum_{n} \theta_{n}^{(\mathrm{m})} \mathrm{e}^{\mathrm{j} K_{n} z},
\end{aligned}
$$

where $K_{n}=\frac{n \pi}{d}$. The solution of (5) can be written in the form

$$
\begin{aligned}
& S_{5}(z)=-\sum_{n} G_{n}(z)\left[e_{15} \theta_{n}^{(\mathrm{e})} E_{1}+q_{15} \theta_{n}^{(\mathrm{m})} H_{1}\right], \\
& G_{n}(z)=\frac{K_{n}^{2}}{\rho \omega^{2}-C_{55} K_{n}^{2}} \mathrm{e}^{\mathrm{j} K_{n} z} .
\end{aligned}
$$

Combining (7) with (1) gives

$$
\begin{aligned}
D_{1} & =\left[\left(\varepsilon_{11}(z)-e_{15}^{2} \theta_{n}^{(\mathrm{e})} G^{\mathrm{S}}\right) E_{1}-e_{15} q_{15} \theta_{n}^{(\mathrm{m})} G^{\mathrm{S}} H_{1}\right] \\
& \times \theta^{(\mathrm{e})}(z)=\varepsilon_{\perp}(z) E_{1}+\alpha_{11}(z) H_{1}, \\
B_{1} & =\left[\left(\mu_{11}(z)-q_{15}^{2} \theta_{n}^{(\mathrm{m})} G\right) H_{1}-e_{15} q_{15} \theta_{n}^{(\mathrm{e})} G E_{1}\right] \theta^{(\mathrm{m})} \\
& =\mu_{\perp}(z) H_{1}+\beta_{11}(z) E_{1}, \\
G & =\sum_{n} G_{n}(z) .
\end{aligned}
$$

When $\lambda \gg d$, we can approximate it by $G=G_{1}(n=$ 1 ). In such a case, the space average values of all material coefficients are applicable for the effective medium, i.e., 


$$
\boldsymbol{w}=\frac{1}{2 d} \int_{2 n d}^{2(n+1) d} \boldsymbol{w}(z) \mathrm{d} z,
$$

where $\boldsymbol{w}=(\boldsymbol{C}, \boldsymbol{\varepsilon}, \boldsymbol{\mu}, \boldsymbol{e}, \boldsymbol{q}, \boldsymbol{\alpha}, \boldsymbol{\beta})$.

Moreover, if the frequency of the incident EM wave is near to the fundamental resonance frequency, by ignoring the other high-order reciprocal vectors and taking into account the boundary conditions, we can get

$$
\begin{aligned}
D_{1} & =\left(\varepsilon_{11}-G e_{15}^{2}\right) E_{1}+G e_{15} q_{15} H_{1} \\
& =\varepsilon_{\perp} E_{1}+G e_{15} q_{15} H_{1}, \\
B_{1} & =\left(\mu_{11}-G q_{15}^{2}\right) H_{1}+G e_{15} q_{15} E_{1} \\
& =\mu_{\perp} H_{1}+G e_{15} q_{15} E_{1}, \\
G & \approx G_{1}=\frac{2}{d^{2} \rho\left(\omega^{2}-\omega_{\mathrm{S}}^{2}\right)} .
\end{aligned}
$$

$\omega_{\mathrm{S}}=G_{1} \nu_{\mathrm{S}}$ and $\nu_{\mathrm{S}}=\sqrt{C_{55} / \rho}$ are the fundamental resonance frequency and the effective velocity of the transverse acoustic wave in the superlattice. Similarly, we can get $D_{2}, B_{2}, D_{3}$ and $B_{3}$.

\section{Dispersion relations for phonon-polariton in uniaxial magnetodielectric superlattices}

The constitutive equation for the effective medium can be described by $[7,8]$ :

$$
\left[\begin{array}{l}
D_{1} \\
D_{2} \\
D_{3} \\
B_{1} \\
B_{2} \\
B_{3}
\end{array}\right]=
$$

$$
\left[\begin{array}{cccccc}
\varepsilon_{\perp} & 0 & 0 & G e_{15} q_{15} & 0 & 0 \\
0 & \varepsilon_{\perp} & 0 & 0 & G e_{15} q_{15} & 0 \\
0 & 0 & \varepsilon_{\|} & 0 & 0 & \alpha_{33} \\
G e_{15} q_{15} & 0 & 0 & \mu_{\perp} & 0 & 0 \\
0 & G e_{15} q_{15} & 0 & 0 & \mu_{\perp} & 0 \\
0 & 0 & \beta_{33} & 0 & 0 & \mu_{\|}
\end{array}\right]\left[\begin{array}{c}
E_{1} \\
E_{2} \\
E_{3} \\
H_{1} \\
H_{2} \\
H_{3}
\end{array}\right] .
$$

In the following, we use the shorthand notation $e_{15}=e$ and $q_{15}=q$.

In the linear approximation, we have

$$
\begin{aligned}
\tilde{B}_{i} & =\mu_{i n}(\boldsymbol{\kappa}) \tilde{H}_{n}, \\
\tilde{D}_{i} & =\varepsilon_{i n}(\boldsymbol{\kappa}) \tilde{E}_{n},
\end{aligned}
$$

where $($ see $[3,7])$

$$
\begin{aligned}
& \mu_{i n}(\boldsymbol{\kappa})=\mu_{i n}^{\mathrm{S}}+q_{i k l} \kappa_{l} \tilde{G}_{j k}(\boldsymbol{\kappa}) \kappa_{p} q_{p j n} \theta^{(\mathrm{m})}, \\
& \varepsilon_{i n}(\boldsymbol{\kappa})=\varepsilon_{i n}^{\mathrm{S}}+e_{i k l} \kappa_{l} \tilde{G}_{j k}(\boldsymbol{\kappa}) \kappa_{p} e_{p j n} \theta^{(\mathrm{e})}, \\
& \alpha_{i k}(\boldsymbol{\kappa})=e_{i k l} \kappa_{j} \tilde{G}_{n l}(\boldsymbol{\kappa}) q_{j l n} \theta^{(\mathrm{e})} \theta^{(\mathrm{m})} .
\end{aligned}
$$

According to $[4-6]$ as a rule the connection $\boldsymbol{\beta}=\boldsymbol{\alpha}^{\mathrm{T}}$ holds.

The Fourier transformed dynamic elastic Green function has the general form [3]:

$$
\tilde{G}_{j k}(\boldsymbol{\kappa})=\frac{\kappa^{2}}{\rho \omega^{2}-C_{i j k l} \kappa_{k} \kappa_{l}},
$$

where $\boldsymbol{\kappa}=\boldsymbol{k}+\boldsymbol{K}$. Using the notation for averaged medium $\theta^{(m, p)}=\theta_{n}^{(m, p)}(\kappa=0)$ we can write

$$
\begin{aligned}
& D_{1}=\varepsilon_{\perp} E_{1}+G e q H_{1}, \quad B_{1}=G e q E_{1}+\mu_{\perp} H_{1}, \\
& D_{2}=\varepsilon_{\perp} E_{2}+G e q H_{2}, \quad B_{2}=G e q E_{2}+\mu_{\perp} H_{2}, \\
& D_{3}=\varepsilon_{\|} E_{3}+\alpha_{33} H_{3}, \quad B_{3}=\beta_{33} E_{3}+\varepsilon_{\|} H_{3} .
\end{aligned}
$$

The divergence Maxwell equations then read

$$
\begin{gathered}
\nabla \cdot \boldsymbol{D}=k_{1}\left(\varepsilon_{\perp} E_{1}+G e q H_{1}\right) \\
\quad+k_{3}\left(\varepsilon_{\|} E_{3}+\alpha_{33} H_{3}\right)=0, \\
\nabla \cdot \boldsymbol{B}=k_{1}\left(G e q E_{1}+\mu_{\perp} H_{1}\right) \\
\quad+k_{3}\left(\beta_{33} E_{3}+\mu_{\|} H_{3}\right)=0 .
\end{gathered}
$$

\section{The oblique incidence case}

For the oblique incident wave (angle $\theta$ ) after some manipulations, we obtain

$$
E_{3}=-\left(a E_{1}+e H_{1}\right) \frac{s}{c}, \quad H_{3}=-\left(b H_{1}+d E_{1}\right) \frac{s}{c},
$$

where

$$
\begin{array}{rr}
a=\left(\varepsilon_{\perp} \mu_{\perp}+G e q \beta_{33}\right) L, & b=\left(G e q \alpha_{33}+\varepsilon_{\perp} \mu_{\perp} \mu_{\|}\right) L, \\
d=\mu_{\perp}\left(G e q+\mu_{\|} \beta_{33}\right) L, & e=\left(\varepsilon_{\perp} \mu_{\perp} \mu_{\|}-\varepsilon_{\|} \alpha_{33}\right) L
\end{array}
$$

with $L=\left(\beta_{33} \alpha_{33}-\varepsilon_{\perp} \mu_{\perp}\right)^{-1}$, and we have denoted

$$
c=\cos \theta, \quad s=\sin \theta .
$$

Using (24) in the rotational part of Maxwell's equations, we obtain

$$
\left[\begin{array}{cccc}
s^{2} a / c+c & s^{2} e / c & -G \nu e q & -\nu \mu_{\|} \\
-s^{2} d / c & s^{2} b / c+c & \nu \varepsilon_{\|} & G \nu e q \\
G \nu e q & \nu \mu_{\|} & c & 0 \\
-\nu \varepsilon & -G \nu e q & 0 & c
\end{array}\right]\left[\begin{array}{c}
E_{1} \\
H_{1} \\
E_{2} \\
H_{2}
\end{array}\right]=0 .
$$

Solution condition of (28) gives the dispersion relation

$$
\begin{aligned}
& \eta^{2} \nu^{4}+\nu^{2} \eta\left[2 c^{2}+s^{2}(a+d)\right] \\
& \quad+c^{-1}\left(s^{2} a+c^{2}\right)\left(s^{2} b+c^{2}\right)+s^{4} e d=0
\end{aligned}
$$

with $\eta=\eta(\omega)=(G e q)^{2}-\varepsilon_{\perp} \mu_{\perp}$.

For the case of normal EM wave incidence $(c=1$, $s=0)$ the above equation takes much simpler form

$$
\eta^{2} \nu^{4}+\nu^{2} \eta+1=0 \text {. }
$$

Equation (30) describes a polariton behaviour given by strong frequency dependence of $\eta(\omega)$ near the resonance. To interprete Eq. (29) it is of interest to note that it has algebraic form of the two-square equation

$$
\eta^{2} \nu^{4}+\nu^{2} \eta(A+B)+A B+C=0,
$$

which can be presented by means of the formula

$$
\left(\eta \nu^{2}+A\right)\left(\eta \nu^{2}+B\right)+C=0 .
$$

It can be treated as describing two polariton modes given by vanishing brackets, i.e.

$$
\eta \nu^{2}+A=0, \quad \eta \nu^{2}+B=0
$$

being coupled with each other due to the term $C(\omega)=$ $s^{4} e d$. 
This indicates a specific mechanism for the coupling, being strongly dependent on the direction of the incident EM wave. So, we see that with the oblique EM wave incidence it is possible to manipulate polariton dynamics parameters through varying the incidence angle $\theta$.

\section{Remarks on current and prospective applications of microwave phonon polaritons}

In general, polariton devices will have very low power consumption and be ultrafast. They could also be assembled in parallel into large matrices of thousands of devices each, which would dramatically reduce the cost per device $[1,2,8]$.

Many devices operate through the interaction of radio-frequency ( $\mathrm{RF}$ ) electromagnetic waves with materials. The characterization of the interface and interaction between fields and materials is a critical task in any electromagnetic device or measurement instrument development, from nanoscale to larger scales. Electromagnetic waves in the radio-frequency range have unique properties. These attributes include the ability to travel in guided wave structures, the ability of antennas to launch waves that carry information over long distances, possess measurable phase and magnitude, the capability for imaging and memory storage, dielectric heating, and the ability to penetrate materials.

Some of the applications are related to areas in microelectronics, bioelectromagnetics, homeland security, nanoscale and macroscale probing, magnetic memories, dielectric nondestructive sensing, radiometry, dielectric heating, and microwave-assisted chemistry. For nanoscale devices the $\mathrm{RF}$ wavelengths are much larger than the device. In many other applications the feature size may be comparable or larger than the wavelength of the applied field.

It was found [9] that at resonance, the strong photonphonon coupling changes the character of the propagation providing the propagation mode which is neither a pure photon mode, nor a pure transverse phonon mode.

Periodic structure, being able to provide Rabi's type crossing in the EM waves dispersion, is generally called the electromagnetic band-gap (EBG) medium. It can be one-dimensional (1D), two-dimensional (2D) or three-dimensional (3D). 3D EBGs being the closest to the 3D case, well illustrated by the electron behavior in semiconductors, are rather complicated for both simulation and construction.

Important specific properties of the EBGs provide defects, disturbing the periodicity of the structure. In the aspect of propagation of the EM waves, it can be treated as a resonant cavity. In the transmission response, defects can form an isolated and waveguiding $1 \mathrm{D}$ or $2 \mathrm{D}$ mode inside the forbidden band-gap.

Applications of the EBGs can be in the whole EM range: from RF to X-rays. However, the leading are optical and microwave regions, where they have the most applicable results. Both have their specific problems according to the nature of the medium and its interaction with the EM waves.

Microwave EBGs exemplified in this paper have their own specific properties that are essentially different from optical ones.

The case treated above can provide a new field of investigations, and seems to have a considerable applied potential due to its ability for controlling polariton dynamic behavior, and especially its dispersion.

\section{References}

[1] D. Nešić, Microwave Rev. 7, 18 (2001).

[2] Y. Qian, R. Coccioli, D. Sievenpiper, V. Radisic, E. Yablonovitch, T. Itoh, Microwave J. 42, 66 (1999).

[3] J. Kapelewski, Acta Phys. Pol. A 120, 647 (2011).

[4] C.-W. Nan, Phys. Rev. B 50, 6082 (1994).

[5] C.-W. Nan, M.I. Bichurin, S. Dong, D. Viehland, G. Srinivasan, J. Appl. Phys. 103, 31101 (2008).

[6] G. Srinivasan, Anu. Rev. Mater. Res. 40, 153 (2010).

[7] J. Zhao, R.-C. Yin, T. Fan, M.-H. Lu, C.-L. Du, Y.-F. Chen, Y.-Y. Zhu, S.-N. Zhu, N.-B. Ming, Phys. Rev. B 77, 075126 (2008).

[8] J. Zhao, T. Fan, R.-C. Yin, M.-H. Lu, C.-L. Du, Y.-F. Chen, Y.-Y. Zhu, S.-N. Zhu, N.-B. Ming, Phys. Lett. A 372, 486 (2008).

[9] J. Capmany, B. Ortega, D. Pastor, J. Lightwave Technol. 24, 201 (2006). 